\title{
Resveratrol inhibits proliferation and migration through SIRT1 mediated post-translational modification of PI3K/AKT signaling in hepatocellular carcinoma cells
}

\author{
RONGFEI CHAI, HUILING FU, ZHAODI ZHENG, TINGTING LIU, SHUHUA JI and GUORONG LI \\ Shandong Provincial Key Laboratory of Animal Resistant Biology, School of Life Sciences, \\ Shandong Normal University, Jinan, Shandong 250014, P.R. China
}

Received March 30, 2017; Accepted September 12, 2017

DOI: $10.3892 / \mathrm{mmr} .2017 .7612$

\begin{abstract}
Resveratrol (RES), a polyphenolic compound present in grapes and red wine, has potential anticancer properties. The present study aimed to examine the effects of resveratrol and its underlying mechanism on hepatocellular carcinoma (HCC) cell lines HepG2, Bel-7402 and SMMC-7721. It was demonstrated that resveratrol inhibited the viability and proliferation of HCC cells assessed by MTT and EdU assays. TUNEL assay revealed that resveratrol induced cell apoptosis by increasing HCC apoptosis rate from $3 \pm 0.78 \%$ to $16 \pm 1.12 \%$ with upregulation of B-cell lymphoma (Bcl)-2 associated $\mathrm{X}$, apoptosis regulator and cleaved-poly (ADP-Ribose) polymerase 1 (PARP), and downregulation of Bcl-2, caspase-3, caspase-7 and PARP. As a sirtuin (SIRT) 1 activator, resveratrol elevated SIRT1 protein expression and its enzyme activity and decreased expression levels of phosphorylated (p)-phosphoinositide-3-kinase (PI3K), p-AKT Serine/Threonine Kinase 1 (AKT), and its downstream target p-Forkhead Box O3a in HepG2 cells. Furthermore, inhibition of SIRT1 enzymatic activity by EX527 resulted in increased phosphorylation levels of PI3K and AKT. This demonstrated that resveratrol inhibited the PI3K/AKT pathway by SIRT1 activation. In addition to inhibition of cancer cell migration, tumor suppressor gene DLC1 Rho GTPase activating protein level was upregulated and its phosphorylation was enhanced by AKT with resveratrol treatment. These findings suggested that resveratrol inhibits proliferation and migration through SIRT1 mediated post-translational modification of PI3K/AKT pathway in HCC cells.
\end{abstract}

Correspondence to: Professor Guorong Li, Shandong Provincial Key Laboratory of Animal Resistant Biology, School of Life Sciences, Shandong Normal University, 88 East Wenhua Road, Jinan, Shandong 250014, P.R. China

E-mail: grli@sdnu.edu.cn

Key words: resveratrol, SIRT1, PI3K/AKT, DLC1

\section{Introduction}

Hepatocellular carcinoma (HCC) is one of the major leading causes of tumor-associated deaths, with high rates of incidence and disease-related mortality and morbidity in the world (1). As it is still difficult to make an early diagnosis for HCC, most of the patients are diagnosed at advanced stages. Despite the improvement of conventional therapies for HCC, including surgery, chemotherapy and radiotherapy, the length or quality of life of patients with HCC is still poor. Therefore, it is urgent to develop a new preventive strategy for liver cancer.

Resveratrol (RES, trans-3,5,4'-trihydroxystilbene) is a polyphenol compound derived from grapes, berries, peanuts and other sources, and it has inhibitory effects on several types of cancer cell lines such as colon, lung and prostate and affects diverse molecular targets (2). Sirtuin 1 (SIRT1) has been reported to be a key target of resveratrol in several tumor models $(3,4)$. Whether SIRT1 as a tumor promoter or tumor suppressor remains controversial, it might depend on tumor type (5). Resveratrol suppresses tumor cell growth and metastasis in colorectal cancer cells by targeting SIRT1 protein and regulating NF- $\mathrm{NB}$ signaling pathway (6). The forkhead box $\mathrm{O}$ transcription factors (FoxOs) have emerged as critical transcriptional factors in regulating metabolism and stress responses and been considered as downstream targets of SIRT1. FoxO1 translocated into nucleus increases FoxO1-DNA binding and expression of proapoptotic gene Bim (7). SIRT1 might regulate cell apoptosis by deacetylating FoxOs protein.

The phosphatidylinositol 3'-kinase (PI3K)/AKT pathway plays an important role in cell survival and PI3K activity has been linked to a variety of human cancers (8). AKT, a downstream kinase of PI3K, regulates many cellular proteins including metabolism, apoptosis and proliferation (9). PI3K pathway phosphorylates FoxOs via activation of its downstream kinase AKT (10). Inhibition of PI3K pathway leads to dephosphorylation and nuclear translocation of active FoxOs, which induce cell cycle arrest and apoptosis (11). These indicate that FoxOs are important downstream effectors of PI3K/AKT pathway. Resveratrol has been shown to inhibit activation of multiple survival pathways including PI3K/AKT pathway to induce apoptosis in various cancer cells $(9,12)$. 
Deleted in liver cancer 1 (DLC1), a focal adhesion protein, is identified as a putative tumor suppressor in HCC in 1998 (13). It functions as a RhoGTPase activating protein (RhoGAP) (14). Activated protein kinase $\mathrm{C}$ (PKC) and protein kinase D (PKD) stimulate the association between DLC1 and 14-3-3 protein, which blocks DLC1 nucleocytoplasmic shuttling and inhibits RhoGAP activity of DLC1 (15). DLC1 activity could be regulated by post-translational modification and it might be a substrate of AKT. Expression of DLC1 suppresses cell proliferation, anchorage-independent growth, tumorigenicity and invasiveness in HCC cells (16). DLC1 inhibits Rho-dependent stress fiber formation in fibroblasts and serves as a tumor suppressor gene in human non-small cell lung carcinomas (17). Thus, we hypothesized that AKT involved in regulation of DLC1 mediated cell motility inhibition in HCC.

The purposes of the present study were to determine the molecular mechanism of resveratrol affected proliferation and migration through SIRT1 mediated post-translational modification of PI3K/AKT signaling pathway in HCC cells.

\section{Materials and methods}

Cell culture. The human hepatocellular carcinoma (HCC) cell lines Bel-7402, SMMC-7721, hepatoblastoma cells HepG2 (The HepG2 cell line was originally thought to be a hepatocellular carcinoma cell line but was later shown to be from an hepatoblastoma, PubMed=19751877), and human liver normal cell line HL-7702 were obtained from the Cell Bank of Type Culture Collection of Chinese Academy of Science (Shanghai, China). HepG2 cells were cultured in DMEM, other cells in RPMI 1640 medium. All the experiments were performed in medium containing $10 \%$ fetal bovine serum, $100 \mathrm{U} / \mathrm{ml}$ penicillin and $100 \mu \mathrm{g} / \mathrm{ml}$ streptomycin, maintained at $37^{\circ} \mathrm{C}$ in humidified atmosphere with $5 \% \mathrm{CO}_{2}$.

Proliferation assay by MTT and EdU. MTT assay was used to assess cell viability. The cells were seeded in 96-well plates at a density of $1 \times 10^{4} /$ well overnight and treated without or with resveratrol (Sigma, St. Louis, MO, USA) dissolved in $0.1 \%(\mathrm{v} / \mathrm{v}) \mathrm{DMSO}$ at various concentrations for $24 \mathrm{~h}$. Then cells were incubated with MTT solution for $4 \mathrm{~h}$. The formazan crystals dissolved by $150 \mu \mathrm{l}$ DMSO, the solution was absorbed at $492 \mathrm{~nm}$ using enzyme-linked immunosorbent assay reader (Awareness, Palm City, FL, USA).

Cell proliferation was tested by EdU (5-ethynyl-2-deoxyuridine) incorporation assay kit (Ribobio, Guangzhou, China). Briefly, cells cultured in 96-well plates exposed to $50 \mu \mathrm{M}$ EdU for $2 \mathrm{~h}$ at $37^{\circ} \mathrm{C}$, and fixed in $4 \%$ formaldehyde. After permeabilization with $0.5 \%$ Triton-X, the cells were reacted with 1xApollo reaction cocktail for $30 \mathrm{~min}$, the DNA contents were stained with Hoechst 33342 and visualized under fluorescent microscope. Cells were counted in five selected arbitrarily fields, at least 300 cells were counted per well. EdU positive cells were calculated with (EdU incorporated-in cells/Hoechst stained cells) x100\%.

Apoptosis detection by TUNEL assay. TUNEL staining was performed using an EdUTP TUNEL cell detection kit (Ribobio, Gangzhou, China) according to the manufacturer's instructions. The cells cultured in 96-well plates were treated without or with $100 \mu \mathrm{M}$ resveratrol, fixed in $4 \%$ paraformaldehyde, permeabilized with $0.1 \%$ Triton X-100, washed twice, incubated with TUNEL detecting liquid for $1 \mathrm{~h}$ at $37^{\circ} \mathrm{C}$ and observed by a fluorescent microscope (Olympus, Tokyo, Japan) at $488 \mathrm{~nm}$ excitation and $530 \mathrm{~nm}$ emission. TUNEL positive cells were calculated as the number of apoptotic cells/DAPI stained cells $\times 100 \%$.

Western blotting and Co-immunoprecipitation analysis. The cells were lysed by RIPA (Beyotime, Shanghai, China). The inhibitor of SIRT1, EX-527 (Selleck Chemicals, Houston, TX, USA), was used to PI3K/AKT pathway. Proteins were separated by SDS-PAGE and transferred on membranes were incubated in primary antibodies against SIRT1, p-AKT, AKT, p-PI3K, PI3K, PARP, Cleaved-PARP, p-FoxO3a, Caspase-3/-7, Bax, Bcl-2 and p53 (CST, Danvers, MA, USA) and FoxO1, FoxO3a (Santa Cruz Biotechnology, San Diego, CA, USA) overnight at $4{ }^{\circ} \mathrm{C}$, followed by incubation with HRP-conjugated rabbit/mouse secondary antibodies (ZSGB-BIO, Beijing, China). Protein expressions were visualized ECL detection system (Beyotime, Shanghai, China).

Immunoprecipitation (IP) was carried out using Pierce Classic Magnetic IP/Co-IP Kit (Thermo Scientific, Waltham, MA, USA) according to the manufacturer's protocol. The protein lysates were incubated with DLC1 antibody (BD Biosciences, San Jose, CA, USA), and precipitated with Protein A/G Magnetic Agarose at $4^{\circ} \mathrm{C}$. The immunocomplex collected was washed, and the immunoprecipitates were subjected to western blotting and phosphorylation signals were determined using phospho-AKT substrate (PAS) antibody (CST, Danvers, MA, USA).

Wound healing assay. Cells were seeded into 24-well plates $\left(1.0 \times 10^{5}\right.$ cells/well). Sterile pipette tip was used to produce a wound line between cells after the cells grew to $80-90 \%$ confluence and allowed the cells migrated for $24 \mathrm{~h}$. Images were captured and the relative distance traveled by the leading edge from 0 to $24 \mathrm{~h}$ was assessed using Image Pro Plus 6.0 software $(n=5)$.

SIRT1 activity assays. SIRT1 activity was quantified with a SIRT1 Fluorometric Assay Kit (Sigma, St. Louis, MO, USA) according to the manufacturer's protocol. Fluorescence intensities were measured with a microplate fluorometer (excitation wavelength $=360 \mathrm{~nm}$, emission wavelength $=450 \mathrm{~nm}$ ). Experimental values are represented as a percentage of control.

Statistical analysis. All of the assays were performed three times independently at least. Value presented as the means \pm standard deviation (SD) by GraphPad Prism software (GraphPad Software, CA, USA). Statistical analyses were performed using one-way ANOVA and Student's t-test, ${ }^{*} \mathrm{P}<0.05,{ }^{* *} \mathrm{P}<0.01$ were considered to indicate a statistically significant difference.

\section{Results}

Effect of resveratrol on cell viability and proliferation. Three HCC cell viability was determined by MTT assay. The 

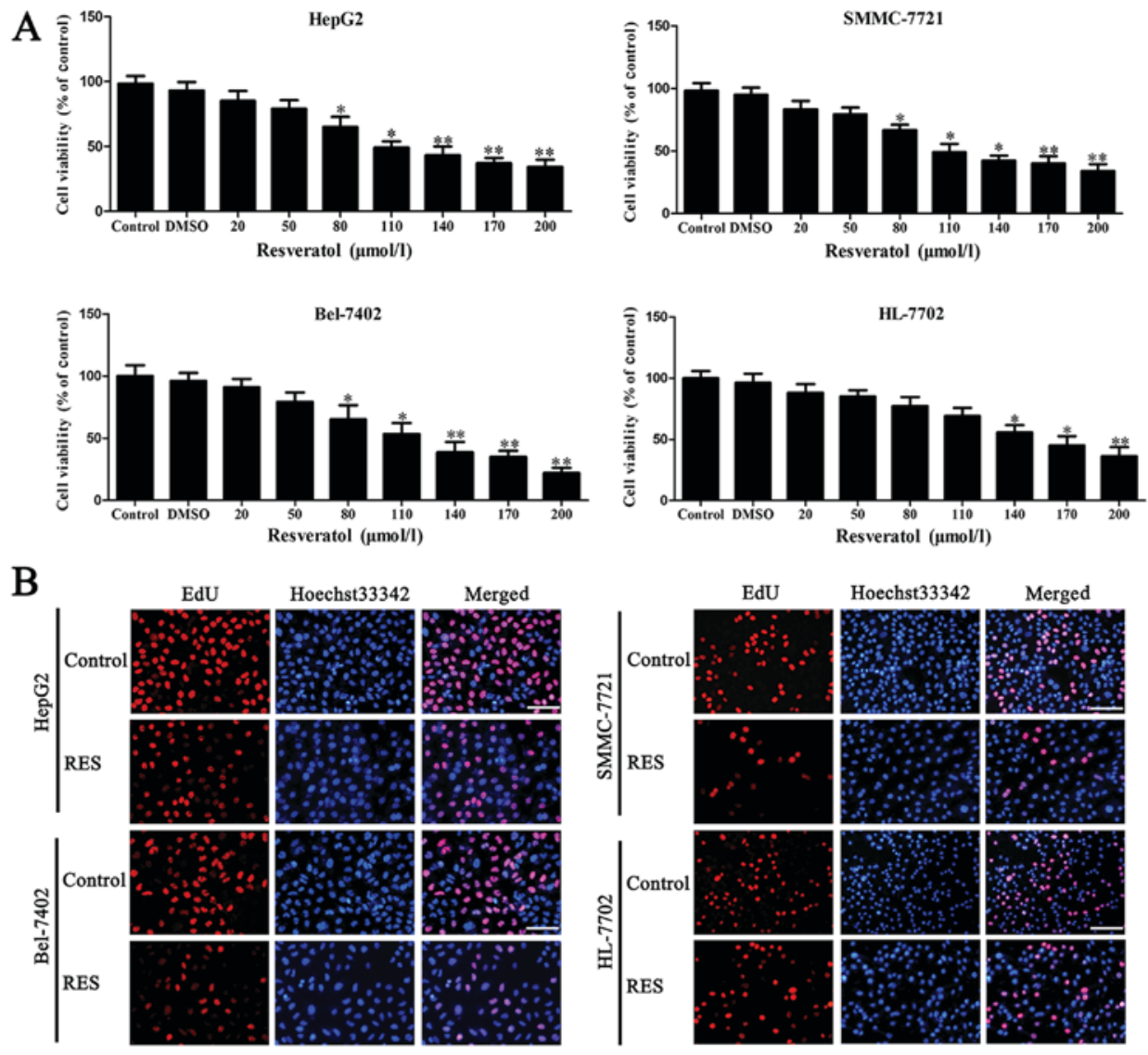

Merged

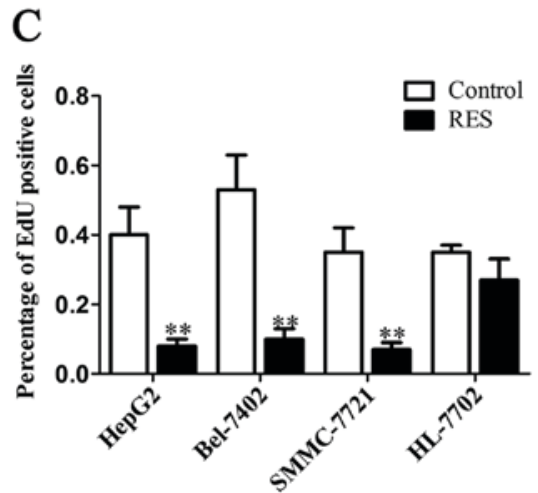

D

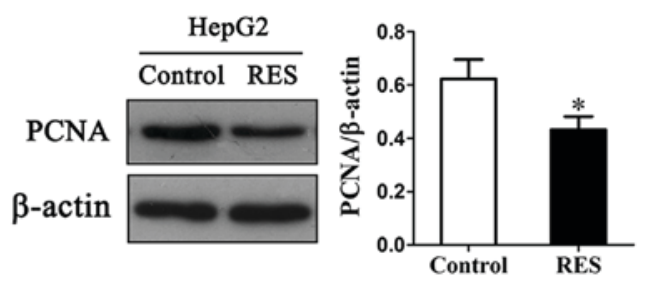

Figure 1. Inhibition of resveratrol on cell viability and proliferation. (A) Selection of resveratrol doses. (B) EdU assay with resveratrol treatment. EdU stain showed red light while Hoechst33342 blue light. Scale bar is $100 \mu \mathrm{m}$. (C) Quantitative analysis of EdU positive cells. (D) Expression of PCNA and relative expression level. ${ }^{*} \mathrm{P}<0.05$ and ${ }^{* *} \mathrm{P}<0.01$ vs. control.

results showed that resveratrol inhibited cell viability when its concentrations were higher than $80 \mu \mathrm{M}$ compared to normal HL-7702 cell (Fig. 1A). From 80-200 $\mu \mathrm{M}, 100 \mu \mathrm{M}$ resveratrol was selected as IC50 (half maximal inhibitory concentration) in the subsequent experiments. It implicated that resveratrol was able to reduce cancer cell viability in a dose-dependent manner. The percentage of EdU positive cells was markedly reduced in $\mathrm{HCC}$ cells with $100 \mu \mathrm{M}$ resveratrol treatment compared to the controls, while non-tumorigenic cell line HL-7702 had a slight reduction (Fig. 1B and C).

To understand the molecular basis of proliferation inhibition caused by resveratrol, proliferation regulation protein PCNA (proliferating cell nuclear antigen) was evaluated in HepG2 cells. The level of PCNA reduced after $100 \mu \mathrm{M}$ resveratrol treatment (Fig. 1D). It was consistent with above results of EdU assay. These findings demonstrate the anti-proliferative effect of resveratrol on HCC cells.

Resveratrol induced apoptosis via decreasing phosphorylation of FoxO3a with suppressing PI3K/AKT pathway. TUNEL assay was assessed whether anti-proliferative effects of resveratrol against $\mathrm{HCC}$ cells are mediated via apoptosis. Results showed that resveratrol increased apoptosis from $4 \pm 0.83 \%$ to $13 \pm 1.32 \%, 3 \pm 0.78 \%$ to $14 \pm 0.72 \%, 5 \pm 0.33 \%$ to $16 \pm 1.12 \%$ in HepG2, Bel-7402 and SMMC-7721 cells (Fig. 2A).

Studies have reported that Bcl-2 could be a crucial target gene of PI3K/AKT signaling, whereas AKT has been shown to negatively regulate the activity of proapoptotic members of the Bcl-2 family (8). Next effects of resveratrol on apoptosis-related proteins were further detected. As showed by 
A
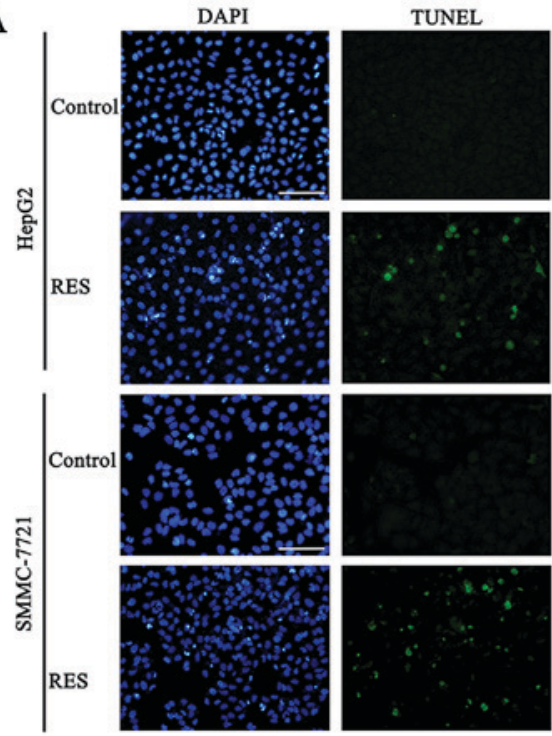

DAPI
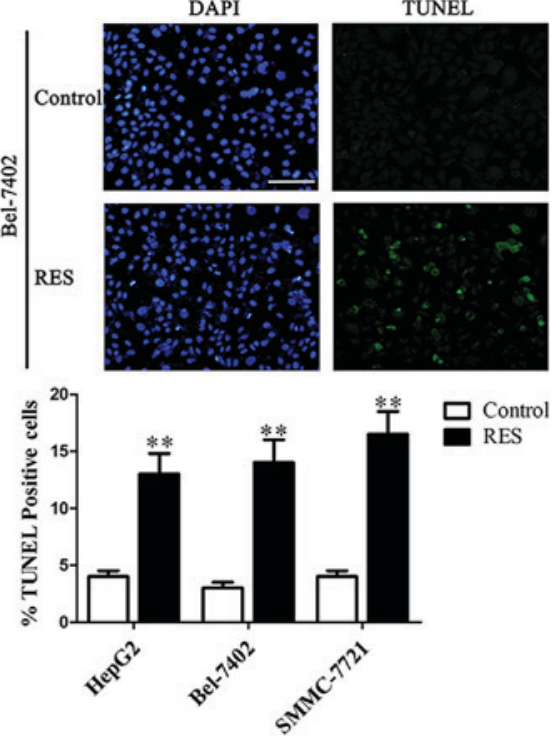

B
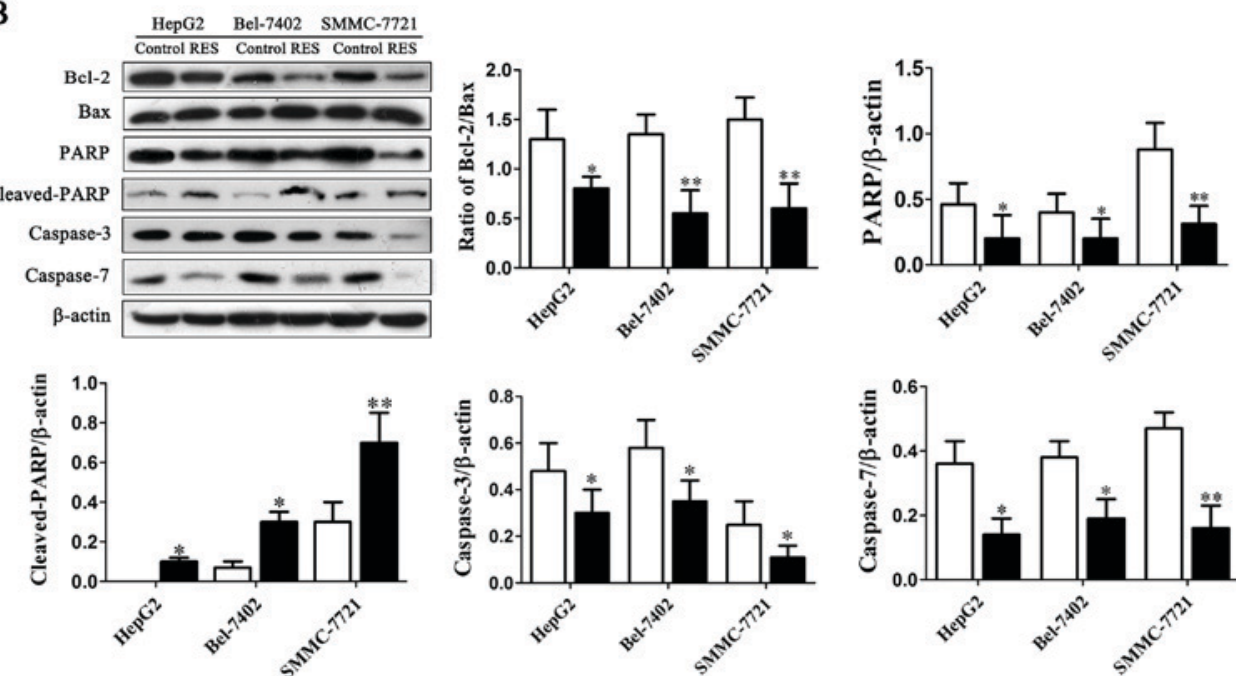

Figure 2. Resveratrol induced apoptosis and regulated apoptosis-related protein levels in liver cancer cells. (A) Fluorescent microscopy images of TUNEL assay. DAPI staining (blue) was used to identify cell nuclei, TUNEL-positive cells are by green. Scale bar represents $100 \mu \mathrm{m}$. Bar graph showed the percentage of apoptotic cells. (B) Protein levels of PARP, cleaved PARP, caspase-3, caspase-7, Bcl-2 and Bax. Bar graphs showed their relative levels to $\beta$-actin, and the ratio of $\mathrm{Bcl}-2 / \mathrm{Bax} .{ }^{*} \mathrm{P}<0.05$ and ${ }^{* *} \mathrm{P}<0.01$ vs. control.

Fig. 2B, resveratrol inhibited Bcl-2 expression and concomitant up-regulated proapoptotic protein Bax, causing a significant decrease in $\mathrm{Bcl}-2 / \mathrm{Bax}$ ratio. The apoptosis regulators were further detected and the precursor forms of caspase-3/7 induced by resveratrol were down-regulated obviously in $\mathrm{HCC}$ cells (Fig. 2B). The activation of caspases were also related to another marker of apoptosis, proteolysis of the DNA repair enzyme PARP (18). The results indicated that precursor form PARP decreased as active form cleavage-PARP significantly enhanced (Fig. 2B).

\section{Resveratrol activated SIRT1 and inhibited SIRT1-mediated} post-translational modification of PI3K/AKT signaling. To investigate effects of resveratrol on the pathway of $\mathrm{PI} 3 \mathrm{~K} / \mathrm{AKT} / \mathrm{FoxO}$ a to induce cell apoptosis, western blotting was performed for their phosphorylation levels. Resveratrol inhibited phosphorylation of PI3K and AKT without effect on total levels of PI3K and AKT in HepG2 cells (Fig. 3D), and inhibited FoxO3a phosphorylation in HCC cells with no total
FoxO3a change (Fig. 3D). These data indicated that resveratrol down-regulated p-FoxO3a level with reduction of phosphorylation level of PI3K/AKT.

We then examined whether resveratrol stimulates expression of SIRT1. Resveratrol up-regulated protein expression of SIRT1 in HCC cells but not in normal liver HL-7702 cells (Fig. 3A). Deacetylation of FoxO proteins has been shown to result from the activity of SIRT1 (19). It has been shown that SIRT1 promotes transcription of FoxO target genes involved in stress resistance, while decreasing transcription of genes involved in apoptosis (20). Our result showed that protein levels of FoxO1 and Ac-FoxO1 were significantly decreased with resveratrol treatment compared with control (Fig. 3D). Up-regulation of SIRT1 activated by resveratrol involved in deacetylation of FoxO1.

To determine the relationship between SIRT1 activity and PI3K/AKT signaling pathway, the activity of intracellular SIRT1 was analyzed after SIRT1 inhibitor EX527 was used. Consistent with its protein level, SIRT1 activity increased by 
A
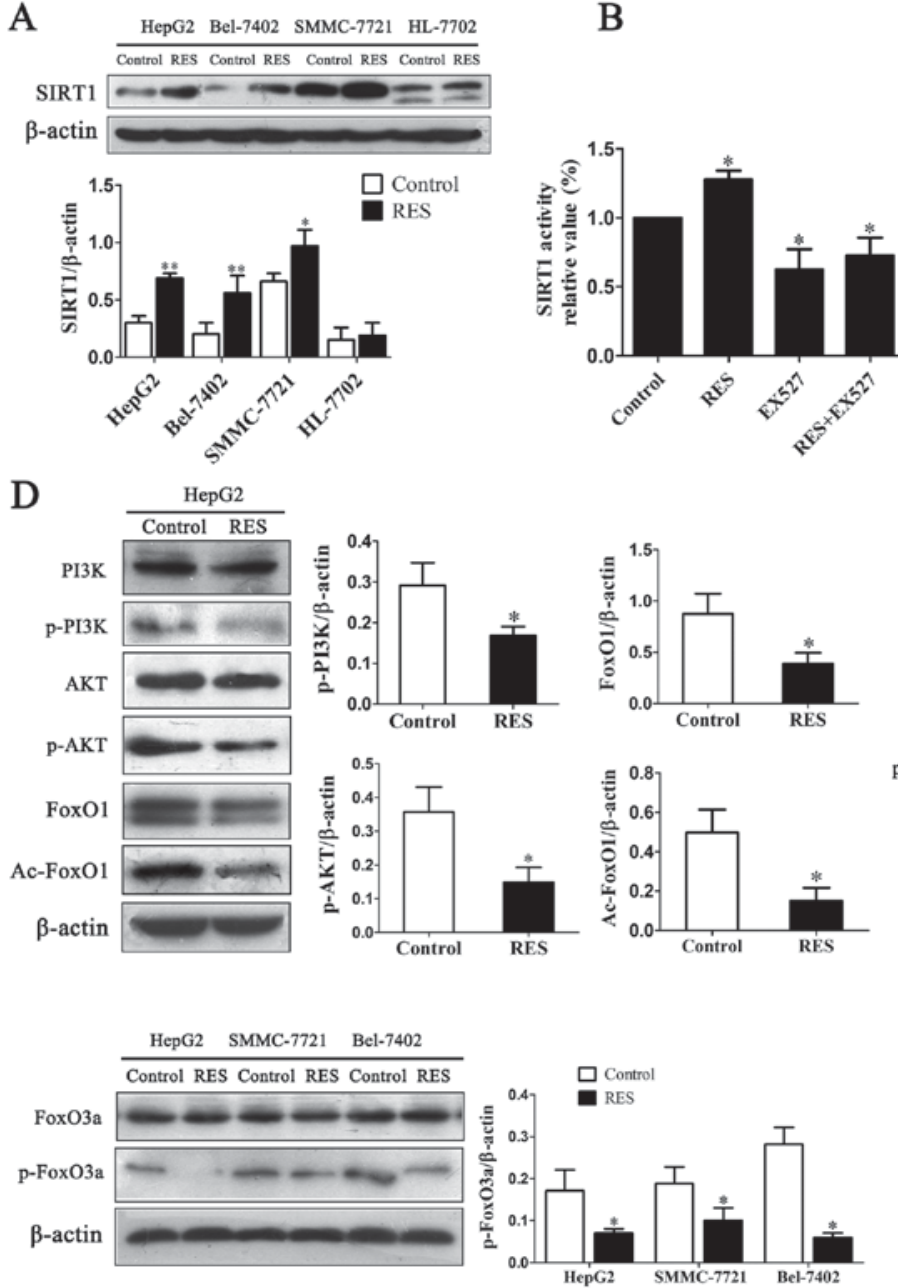
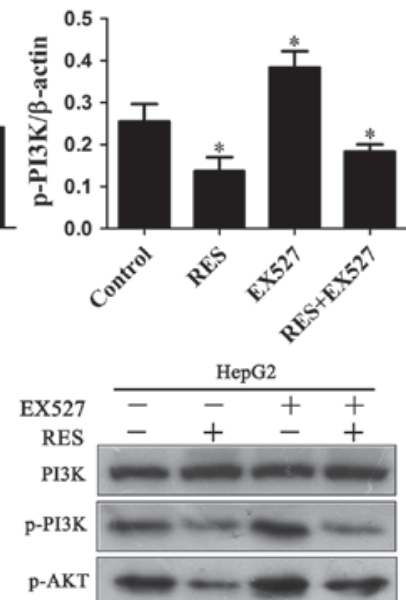

C

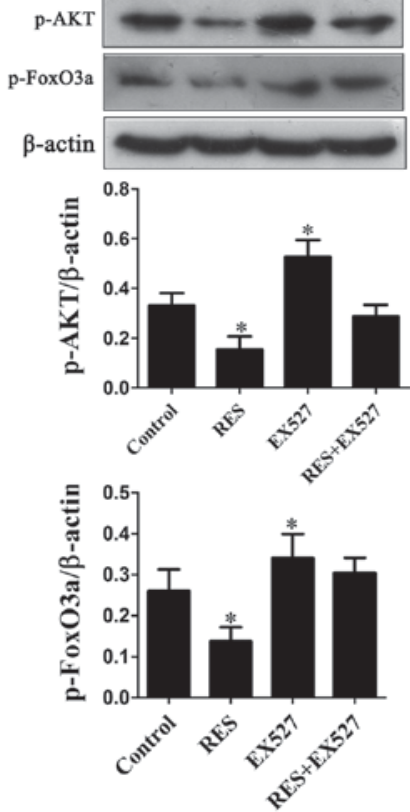

Figure 3. (A) Resveratrol significantly activated protein expression of SIRT1 in liver cancer cells. (B) EX527 inhibited deacetylase activity of SIRT1 in HepG2 cells. (C) Protein levels of PI3K, p-PI3K, p-AKT and p-FoxO3a after treated with EX527. (D) Protein levels of PI3K, p-PI3K, AKT, p-AKT, FoxO1, Ac-FoxO1, FoxO3a and p-FoxO3a. The bar graphs and representative images are shown. ${ }^{*} \mathrm{P}<0.05$ and ${ }^{* *} \mathrm{P}<0.01$ vs. control.

resveratrol and decreased after exposure to EX527 in HepG2 cells (Fig. 3B). Treatment of $1 \mu \mathrm{M}$ EX527 enhanced p-PI3K, p-AKT and p-FoxO3a levels while slight effect on total PI3K (Fig. 3C). These results showed that resveratrol suppressed post-translational modification of SIRT1 mediated PI3K/AKT signaling.

Resveratrol enhanced phosphorylation of DLC1 by AKT and inhibited cell migration. As PI3K/AKT pathway is an important cell survival cascade, DLC1 might be a substrate of AKT. In order to explore whether AKT involved in regulation of tumor suppressor DLC1, phosphorylation on the biological activities of DLC1 was demonstrated in HCC cells. DLC1 protein expression elevated in three kinds of HCC cells and no effect on HL-7702 cells by resveratrol (Fig. 4A). An antibody against PAS (phospho-AKT substrate) was employed to detect phosphorylation of DLC1. Immunoprecipitation result demonstrated that DLC1 phosphorylation level was enhanced by resveratrol in HepG2 cells (Fig. 4B). The relevance and functional effect of AKT mediated phosphorylation of DLC1 remain unclear and await further investigation.

Wound healing assay measures the ability of cells to migrate into an area of a cell culture plate denuded of cells (wound). Our result showed that resveratrol inhibited the wound closures from 32.5 to $11.5 \%$ in HepG2 cells by $24 \mathrm{~h}$ treatment (Fig. 4C). The findings revealed that up-regulation of DLC1 and its phosphorylation level via resveratrol treatment might cause motility inhibition in cancer cells.

\section{Discussion}

Although the capacity of resveratrol to prevent cancer development has been studied for many years, its mechanism underlying remains to be fully elucidated. Proliferating cell nuclear antigen (PCNA) is a critical event in growth regulation of cancer cells (21). The anti-metastatic effect of resveratrol 

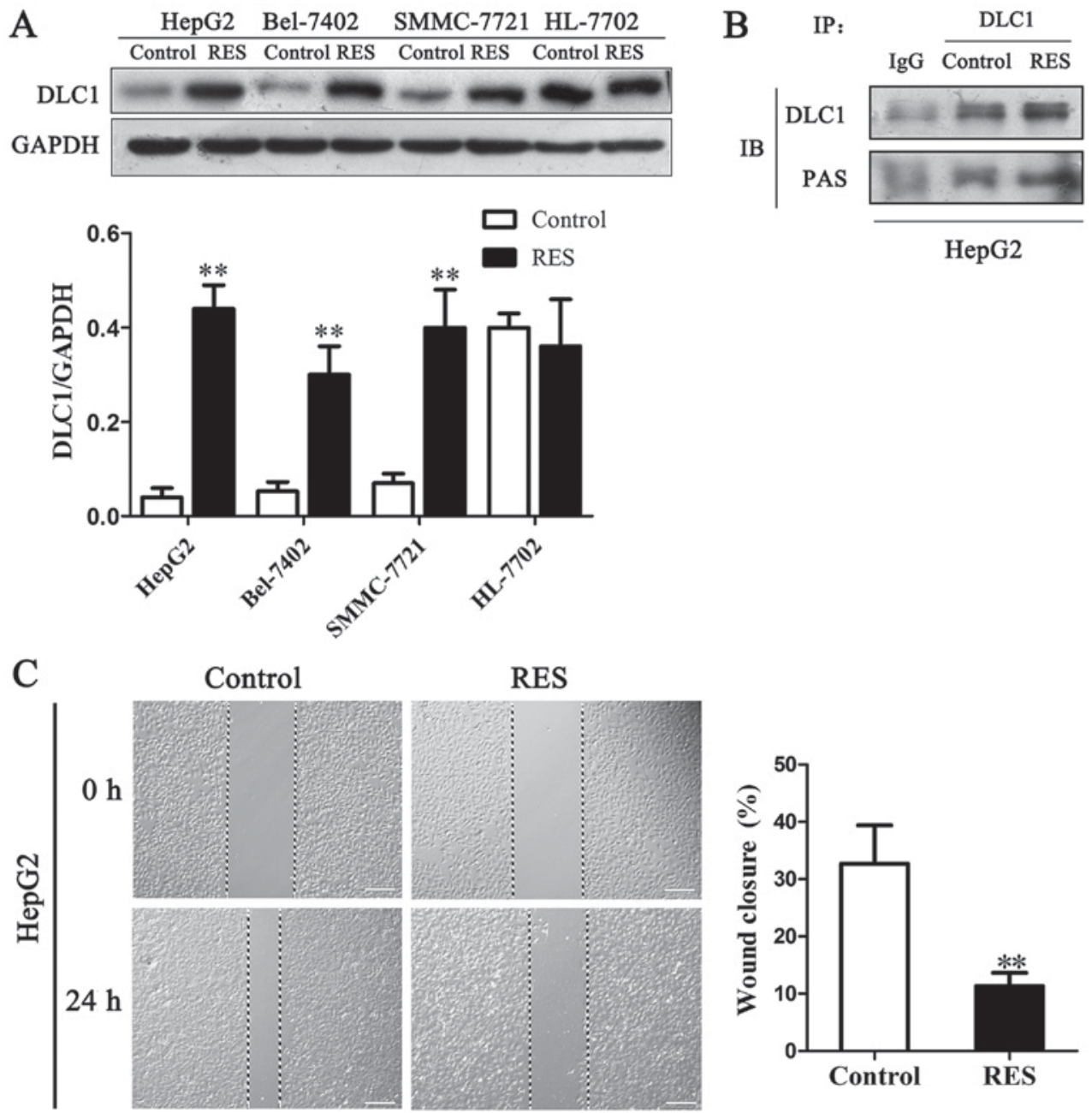

Figure 4. Resveratrol inhibited migration and enhanced phosphorylation of DLC1 by AKT in HepG2 cell. (A) Expression of DLC1 with resveratrol treatment in liver cancer cells and HL-7702. Bar graph showed the ratio of DLC1 protein to GAPDH. (B) DLC1 was immunoprecipitated by DLC1 antibody and subjected to western blotting using PAS antibody. Phosphorylation was elevated with resveratrol treatment. (C) Scratch images and quantitation of wound closure. ${ }^{* *} \mathrm{P}<0.01$ vs. control.

was associated with restriction of invasion, mobility, adhesion, and MMP expression in colon carcinoma (22). Here, we found that resveratrol inhibited the viability and proliferation by MTT and EdU assays and suppressed expression of PCNA accompanying proliferation inhibition in HCC cells.

The PI3K/AKT signaling is a critical pathway in cell proliferation, survival, neovascularization and tumor growth (23). AKT is an important downstream target kinase of PI3K signaling pathway. Activated AKT can inhibit release of cytochrome $\mathrm{c}$ and apoptosis factor, thereby inhibiting apoptosis and promote the growth of cancer cells (8). Resveratrol has been shown to inhibit constitutive activation of PI3K/AKT pathway to induce apoptosis in several types of cancer cells $(12,24)$. FoxO3a is the downstream targets of AKT and AKT can promote FoxO3a phosphorylation, leading to FoxO3a translocation from the nucleus to the cytoplasm, which de-activates FoxO3a; conversely, inhibition of AKT promotes de-phosphorylation of FoxO3a, resulting in nuclear translocation of FoxO3a (25). Our results showed that resveratrol resulted in significant inhibition in constitutively elevated levels of phosphorylated PI3K/AKT and reduced phosphorylated FoxO3a significantly in HepG2 cells. AKT inhibits apoptosis through multiple targets, including Bcl-2 family and caspase proteases (8). Bcl-2 members are well characterized as regulators of apoptosis, such as Bax and Bim. The ratio of $\mathrm{Bcl}-2 / \mathrm{Bax}$ protein regarded as a driving force for apoptosis in cancer cells (26). Caspases are a family of cell death proteases triggered in response to proapoptotic signals and play an essential role in the execution phase of apoptosis (27). TUNEL assays are used to detect DNA fragmentation from apoptosis. In the present study, resveratrol resulted in an increase of green fluorescence signal which was indicative of apoptosis. Also, resveratrol caused a significant down-modulation of Bcl-2/Bax ratio and activated caspase-3, caspase-7, PARP and induced the cleavage-PARP in HCC cells. It suggested that the apoptosis of HCC cells induced by resveratrol might act through the mitochondrial pathways.

SIRT1 plays a key role in both cell death and survival with p53 family members, FoxOs and the nuclear factor- $\kappa \mathrm{B}$ family (28). Furthermore, resveratrol suppresses the proliferation of gastric cancer cells in a SIRT1-dependent manner in vitro and in vivo (29). We showed that resveratrol significantly increased SIRT1 expression in HCC cells. As a nicotinamide adenine dinucleotide-dependent protein deactylase, SIRT1 
is known to be directly involved in the acetylation of FoxOs and expression of proapoptotic protein Bim (19). FoxO1 has emerged as an important protein that modulates the expression of apoptosis-related genes in cancer cells (7). SIRT1 knockdown enhanced Ac-FoxO1 expression to block reactive oxygen species-induced apoptosis in mouse embryonic stem cells (30). Our results showed that resveratrol significantly decreased expressions of FoxO1 and Ac-FoxO1 with activation of SIRT1 by resveratrol.

SIRT1 has been also implicated as a negative regulator for the PI3K/AKT pathway by deacetylating the tumor suppressor PTEN (31) and by down-regulation of both AKT and phosphorylation levels to inhibit the PI3K/AKT pathway in glioblastoma cell (32). The regulation of PI3K/AKT pathway by SIRT1 may provide a potential mechanism in tumorigenesis, and SIRT1 inhibitor EX527 was used to evaluate the underlying mechanism. We found that resveratrol up-regulated SIRT1 level to decrease PI3K and AKT phosphorylation and the phosphorylation of PI3K and AKT became significantly higher when SIRT1 was inhibited in HepG2 cells. It indicated that the inhibition of PI3K/AKT pathway by resveratrol is mediated by up-regulation of SIRT1.

DLC1 is a Rho GTPase-activating protein (RhoGAP) and frequently deleted and underexpressed in cancers (14). Restoration of DLC1 gene expression induces apoptosis and inhibits both cell growth and tumorigenicity in HCC cells (33). Our previous results has been shown that DLC1 is a multifunctional protein which interacts with tensin, talin, FAK in focal adhesion $(34,35)$. DLC1 expression could significantly suppress Rho-dependent actin stress fiber formation in hepatocellular carcinoma and fibroblast cell lines (16). Cell migration is tightly regulated by the activity of Rho proteins through actin cytoskeletal rearrangements (36). In addition, DLC1 overexpression inhibited cell migration by induced disassembly of stress fibers and extensive membrane protrusions around cells on laminin-1 in HCC (37). Our result showed that resveratrol significantly up-regulated expression of DLC1 protein and inhibited the migration ratio from 32.5 to $11.5 \%$ in HCC cells, indicating that induced DLC1 level was associated with tumor suppression effect. The post-translational modification of DLC1 has garnered much attention as the important regulatory mechanism of DLC1 activity, and kinases such as AKT, PKC and PKD have been shown to phosphorylate DLC1 at different residues and regulate its biological activities via RhoGAP-dependent as well as RhoGAP-independent pathways $(15,38)$. Phosphorylation of DLC1 by PKA contributes to enhance RhoGAP activity and promotes activation of DLC1, which suppresses hepatoma cell growth, motility and metastasis both in vitro and in vivo models (39). To elucidate whether AKT could phosphorylate DLC1, an antibody against PAS (phospho-AKT substrate) was employed to detect phosphorylation of DLC1. Our findings showed that DLC1 was directly phosphorylated by AKT in HepG2 cells. These results suggested that DLC1 as a tumor suppressor was up-regulated by resveratrol and its post-translational modification was mediated by PI3K/AKT signaling. Although previous studies have characterized functional effects of the identified phosphorylated residues of DLC1 (40), the physiological stimuli of these phosphorylations remain unclear. Future work are warrant to clarify how DLC1 regulated by its domains and phosphorylation as well as precise downstream mechanisms through post-translational modification of DLC1 acts as a tumor suppressor.

Taken together, our findings suggested that resveratrol activated SIRT1 to induce liver cancer cell apoptosis and to inhibit migration through SIRT1 mediated post-translational regulation of PI3K/AKT signaling and phosphorylation level of FoxO3a and DLC1 and deacetylation of FoxO1 leading to tumor suppression in HCC cells.

\section{Acknowledgements}

This work was supported by the National Natural Science Foundation of China (Grant No. 31672377), the Major Key Science and Technology Project of Shandong Province (2015ZDJS04003), the Key Program of Shandong Provincial Natural Science Foundation of China (ZR2013CZ002), Science and Technology Program of Jinan (201202033).

\section{References}

1. El-Serag HB and Rudolph KL: Hepatocellular carcinoma: Epidemiology and molecular carcinogenesis.Gastroenterology 132: 2557-2576, 2007.

2. Hsieh TC and Wu JM: Resveratrol: Biological and pharmaceutical properties as anticancer molecule. Biofactors 36: 360-369, 2010.

3. Frazzi R, Valli R, Tamagnini I, Casali B, Latruffe N and Merli F: Resveratrol-mediated apoptosis of hodgkin lymphoma cells involves SIRT1 inhibition and FOXO3a hyperacetylation. Int J Cancer 132: 1013-1021, 2013.

4. Li Y, Zhu W, Li J, Liu M and Wei M: Resveratrol suppresses the STAT3 signaling pathway and inhibits proliferation of high glucose-exposed HepG2 cells partly through SIRT1. Oncol Rep 30: 2820-2828, 2013.

5. Fang Y and Nicholl MB: Sirtuin 1 in malignant transformation: Friend or foe? Cancer Lett 306: 10-14, 2011.

6. Buhrmann C, Shayan P, Popper B, Goel A and Shakibaei M: Sirt1 is required for resveratrol-mediated chemopreventive effects in colorectal cancer cells. Nutrients 8: 145, 2016.

7. Fu $\mathrm{Z}$ and Tindall DJ: FOXOs, cancer and regulation of apoptosis. Oncogene 27: 2312-2319, 2008.

8. Song G, Ouyang G and Bao S: The activation of Akt/PKB signaling pathway and cell survival. J Cell Mol Med 9: 59-71, 2005.

9. Liu MH, Lin XL, Li J, He J, Tan TP, Wu SJ, Yu S, Chen L, Liu J, Tian W, et al: Resveratrol induces apoptosis through modulation of the Akt/FoxO3a/Bim pathway in HepG2 cells. Mol Med Rep 13: 1689-1694, 2016.

10. Shankar S, Chen Q and Srivastava RK: Inhibition of PI3K/AKT and MEK/ERK pathways act synergistically to enhance antiangiogenic effects of EGCG through activation of FOXO transcription factor. J Mol Signal 3: 7, 2008.

11. Tzivion G, Dobson M and Ramakrishnan G: FoxO transcription factors; Regulation by AKT and 14-3-3 proteins. Biochim Biophys Acta 1813: 1938-1945, 2011.

12. Hussain AR, Uddin S, Bu R, Khan OS, Ahmed SO, Ahmed M and Al-Kuraya KS: Resveratrol suppresses constitutive activation of AKT via generation of ROS and induces apoptosis in diffuse large B cell lymphoma cell lines. PLoS One 6: e24703, 2011.

13. Yuan BZ, Miller MJ, Keck CL,Zimonjic DB, Thorgeirsson SS and Popescu NC: Cloning, characterization, and chromosomal localization of a gene frequently deleted in human liver cancer (DLC-1) homologous to rat RhoGAP. Cancer Res 58: 2196-2199, 1998.

14. Lahoz A and Hall A: DLC1: A significant GAP in the cancer genome. Genes Dev 22: 1724-1730, 2008.

15. Scholz RP, Regner J, Theil A, Erlmann P, Holeiter G, Jähne R, Schmid S, Hausser A and Olayioye MA: DLC1 interacts with 14-3-3 proteins to inhibit RhoGAP activity and block nucleocytoplasmic shuttling. J Cell Sci 122: 92-102, 2009.

16. Wong CM, Yam JW, Ching YP, Yau TO, Leung TH, Jin DY and $\mathrm{Ng}$ IO: Rho GTPase-activating protein deleted in liver cancer suppresses cell proliferation and invasion in hepatocellular carcinoma. Cancer Res 65: 8861-8868, 2005. 
17. Yuan BZ, Jefferson AM, Baldwin KT, Thorgeirsson SS, Popescu NC and Reynolds SH: DLC-1 operates as a tumor suppressor gene in human non-small cell lung carcinomas. Oncogene 23: 1405-1411, 2004.

18. Communal C, Sumandea M, de Tombe P, Narula J, Solaro RJ and Hajjar RJ: Functional consequences of caspase activation in cardiac myocytes. Proc Natl Acad Sci USA 99: 6252-6256, 2002.

19. Brunet A, Sweeney LB, Sturgill JF, Chua KF, Greer PL, Lin Y, Tran H, Ross SE, Mostoslavsky R, Cohen HY, et al: Stress-dependent regulation of FOXO transcription factors by the SIRT1 deacetylase. Science 303: 2011-2015, 2004.

20. Greer EL and Brunet A: FOXO transcription factors at the interface between longevity and tumor suppression. Oncogene 24 $7410-7425,2005$

21. Zhao H, Ho PC, Lo YH, Espejo A, Bedford MT, Hung MC and Wang SC: Interaction of proliferation cell nuclear antigen (PCNA) with c-Abl in cell proliferation and response to DNA damages in breast cancer. PLoS One 7: e29416, 2012.

22. Wu H, Liang X, Fang Y, Qin X, Zhang Y and Liu J: Resveratrol inhibits hypoxia-induced metastasis potential enhancement by restricting hypoxia-induced factor- 1 alpha expression in colon carcinoma cells. Biomed Pharmacother 62: 613-621, 2008.

23. Engelman JA: Targeting PI3K signalling in cancer: Opportunities, challenges and limitations. Nat Rev Cancer 9: 550-562, 2009

24. Aziz MH, Nihal M, Fu VX, Jarrard DF and Ahmad N: Resveratrol-caused apoptosis of human prostate carcinoma LNCaP cells is mediated via modulation of phosphatidylinositol 3'-kinase/Akt pathway and Bcl-2 family proteins. Mol Cancer Ther 5: 1335-1341, 2006.

25. Liu MH, Yuan C, He J, Tan TP, Wu SJ, Fu HY, Liu J, Yu S, Chen YD, Le QF, et al: Resveratrol protects PC12 cells from high glucose-induced neurotoxicity via PI3K/Akt/FoxO3a pathway. Cell Mol Neurobiol 35: 513-522, 2015.

26. Niquet J and Wasterlain CG: Bim, Bad and Bax: A deadly combination in epileptic seizures. J Clin Invest 113: 960-962, 2004

27. Kaushal GP, Kaushal V, Hong X and Shah SV: Role and regulation of activation of caspases in cisplatin-induced injury to renal tubular epithelial cells. Kidney Int 60: 1726-1736, 2001.

28. Blander G and Guarente L: The Sir2 family of protein deacetylases. Annu Rev Biochem 73: 417-435, 2004

29. Yang Q, Wang B, Zang W, Wang X, Liu Z, Li W and Jia J: Resveratrol inhibits the growth of gastric cancer by inducing G1 phase arrest and senescence in a Sirt1-dependent manner. PLoS One 8: e70627, 2013

30. Chae HD and Broxmeyer HE: SIRT1 deficiency downregulates PTEN/JNK/FOXO1 pathway to block reactive oxygen species-induced apoptosis in mouse Embryonic stem cells. Stem Cells Dev 20: 1277-1285, 2011
31. Ikenoue T, Inoki K, Zhao B and Guan KL: PTEN acetylation modulates its interaction with PDZ domain. Cancer Res 68: 6908-6912, 2008.

32. Wang G, Wang JJ, To TS, Zhao HF and Wang J: Role of SIRT1-mediated mitochondrial and Akt pathways in glioblastoma cell death induced by Cotinus coggygria flavonoid nanoliposomes. Int J Nanomedicine 10: 5005-5023, 2015.

33. Zhou X, Thorgeirsson SS and Popescu NC: Restoration of DLC-1 gene expression induces apoptosis and inhibits both cell growth and tumorigenicity in human hepatocellular carcinoma cells. Oncogene 23: 1308-1313, 2004.

34. Li G, Du X, Vass WC, Papageorge AG, Lowy DR and Qian X: Full activity of the deleted in liver cancer 1 (DLC1) tumor suppressor depends on an LD-like motif that binds talin and focal adhesion kinase (FAK). Proc Natl Acad Sci USA 108: 17129-17134, 2011.

35. Qian X, Li G, Asmussen HK, Asnaghi L, Vass WC, Braverman R, Yamada KM, Popescu NC, Papageorge AG and Lowy DR: Oncogenic inhibition by a deleted in liver cancer gene requires cooperation between tensin binding and Rho-specific GTPase-activating protein activities. Proc Natl Acad Sci USA 104: 9012-9017, 2007.

36. Maddox AS and Burridge $\mathrm{K}$ : RhoA is required for cortical retraction and rigidity during mitotic cell rounding. J Cell Biol 160: 255-265, 2003.

37. Kim TY, Lee JW, Kim HP, Jong HS, Kim TY, Jung M and Bang YJ: DLC-1, a GTPase-activating protein for Rho, is associated with cell proliferation, morphology, and migration in human hepatocellular carcinoma. Biochem Biophys Res Commun 355: 72-77, 2007.

38. Scholz RP, Gustafsson JO, Hoffmann P, Jaiswal M, Ahmadian MR, Eisler SA, Erlmann P, Schmid S, Hausser A and Olayioye MA: The tumor suppressor protein DLC1 is regulated by PKD-mediated GAP domain phosphorylation. Exp Cell Res 317: 496-503, 2011.

39. Ko FC, Chan LK, Sze KM, Yeung YS, Tse EY, Lu P, Yu MH, $\mathrm{Ng}$ IO and Yam JW: PKA-induced dimerization of the RhoGAP DLC1 promotes its inhibition of tumorigenesis and metastasis. Nat Commun 4: 1618, 2013.

40. Hers I, Wherlock M, Homma Y, Yagisawa H and Tavaré JM: Identification of p122RhoGAP (deleted in liver cancer-1) Serine 322 as a substrate for protein kinase B and ribosomal S6 kinase in insulin-stimulated cells. J Biol Chem 281: 4762-4770, 2006. 